\title{
Visual discrimination accuracy for self-initiated stimuli presented during inhalation and exhalation phases: A preliminary study ${ }^{1}$
}

Keita Mizuhara and Hiroshi Nittono

Graduate School of Human Sciences, Osaka University, Japan

*Corresponding Authors

Keita Mizuhara, MSc. E-mail: mizuhara@hus.osaka-u.ac.jp

Hiroshi Nittono, Ph.D. E-mail: nittono@hus.osaka-u.ac.jp

Address: 1-2 Yamadaoka, Suita, Osaka 565-0871, Japan

${ }^{1}$ After conducting this study, it transpired that the experimental procedure contained some methodological issues, rendering the results difficult to interpret. Therefore, we revised the experimental design and conducted a new experiment as a registered report, which has already been published (Mizuhara \& Nittono, 2022). In hindsight, however, the results of both experiments are consistent. It was decided to report the data of this preliminary study for future reference. 


\begin{abstract}
Mizuhara and Nittono (2022) indicated that respiratory phases did not affect the discrimination accuracy of emotional and non-emotional visual stimuli when people voluntarily adjusted the timing of stimulus presentation to their breathing phase. Here we report the result of a preliminary study that was conducted prior to Mizuhara and Nittono (2022). Healthy young adults $(N=28)$ performed an emotional discrimination task consisting of distinguishing fearful vs. neutral facial expressions and a nonemotional discrimination task consisting of distinguishing high- vs. low-contrast Gabor patches during nasal respiration. Participants adjusted the timing of stimulus presentation to the onset of inhalation or exhalation by pressing a stimulus-triggered button. Stimuli were presented for $100 \mathrm{~ms}$ with a 500-ms delay after the button press. It was hypothesized that the discrimination accuracy of choosing fearful facial expressions over neutral ones and choosing high-contrast Gabor patches over low ones would be higher during inhalation than during exhalation. The results indicated that discrimination accuracies did not differ between inhalation and exhalation phases in both tasks. Along with Mizuhara and Nittono (2022), this study provides further evidence that the respiratory phase does not affect the discrimination accuracy of visual stimuli when people voluntarily adjust the timing of stimulus presentation to their breathing.
\end{abstract}

Keywords: Respiratory phase, Nasal respiration, Emotional discrimination, Physical discrimination, Discrimination accuracy 


\section{Introduction}

In their study, Mizuhara and Nittono (2022) asked participants to perform a fear discrimination task (fear vs. neutral facial expressions) and a contrast discrimination task (high-contrast vs. low-contrast Gabor patches). The participants were told to adjust the timing of stimulus presentation by pressing a stimulus-triggered button either just after the onset of inhalation (inhalation-phase condition) or just after the onset of exhalation (exhalation-phase condition). They chose fearful facial expressions over neutral ones or high-contrast Gabor patches over low-contrast ones as accurately as possible. The results indicated that the discrimination accuracy of fearful facial expressions and high-contrast Gabor patches did not differ between inhalation and exhalation.

In this preliminary study, participants performed the same tasks as Mizuhara and Nittono (2022), except for changes in the stimulus intensity step, the interval between pressing the stimulus-triggered button and stimulus presentation, and the order that the trials were conducted in. Stimuli appeared after $500 \mathrm{~ms}$ of participants pressing the stimulus-triggered button.

\section{Method}

The method was mainly the same as in Mizuhara and Nittono (2022), except for the following changes:

\subsection{Participants}

Thirty-six student volunteers who had normal or corrected-to-normal vision participated in the experiment. Data from eight participants were excluded from the analysis according to the predetermined criteria (see Exclusion Criteria). Therefore, the 
final sample was 28 participants (16 men and 12 women) between 21 and 25 years old $(M=22.4, S D=1.1)$. All the participants provided written informed consent before taking part in the study, and received monetary compensation (1,000 Japanese yen, equivalent to about 9.5 US dollars) for their participation. The Behavioral Research Ethics Committee of the Osaka University School of Human Sciences approved this study's protocol (HB019-059). The complete dataset is available at https://osf.io/xz2kb/.

\subsection{Stimuli}

High-intensity (75\% fearful $+25 \%$ neutral) fearful, low-intensity $(25 \%$ fearful $+75 \%$ neutral) fearful, and neutral facial expressions were the same as Mizuhara and Nittono (2022). In addition, a middle-intensity (50\% fearful $+50 \%$ neutral) category was made. Regarding Gabor patches, high-intensity contrast $(\mathrm{RGB}$ values $=[239,239$, $239]$ and $[16,16,16])$, low-intensity contrast $(\operatorname{RGB}$ values $=[207,207,207]$ and $[48$, $48,48])$, and standard contrast $(\operatorname{RGB}$ values $=[191,191,191]$ and $[64,64,64])$ Gabor patches were the same as Mizuhara and Nittono (2022). As well as facial stimuli, middle-intensity contrast $(\operatorname{RGB}$ values $=[223,223,223]$ and $[32,32,32])$ was also added.

\subsection{Procedure}

The experimental settings were similar to Mizuhara and Nittono (2022), except for the following: First, a fixation cross was presented when participants pressed a stimulus-triggered button (i.e., the space bar on a keyboard). Second, the order of the inhalation-phase trials and exhalation-phase trials was alternated. Third, participants were also asked to leave one or more respiratory cycles between trials. Participants could take a short break at the halfway point of each discrimination task. Each task lasted for about 30 minutes. A brief practice session consisting of eight trials was 
conducted before each task.

\subsection{Physiological Recording}

Participants' respiratory movements were recorded by using an air-tube pneumatic sensor connected to a direct-current amplifier (Respiration Belt, Brain Products $\mathrm{GmbH}$, Gilching, Germany). The respiratory sensor was attached around each participant's abdomen with an elastic belt. The signal was recorded by using a V-Amp (Brain Products $\mathrm{GmbH}$, Gilching, Germany) at a sampling rate of 1,000 Hz, with an online filter of DC-200 Hz.

\subsection{Data Reduction}

Respiration data were analyzed by using Brain Vision Analyzer 2 (Brain Products $\mathrm{GmbH}$, Gilching, Germany). A bandpass filter of $0.05-10 \mathrm{~Hz}$ was applied (Schulz et al., 2016). The procedure of detecting inhalation-onset (end-expiratory) troughs and exhalation-onset (end-inspiratory) peaks was the same as Mizuhara and Nittono (2022). Only trials in which the pair of stimuli was presented in appropriate respiratory phases were included in the analysis.

\subsection{Exclusion Criteria}

Any participants who had a history of respiratory or cardiovascular disease were excluded from the analysis. Discrimination accuracy was calculated separately for each participant, stimulus type, stimulus intensity, and respiratory phase. To ensure a reliable number of trials was achieved, the participants' data were analyzed only if each cell contained at least 22 trials (approximately two-thirds of all trials in each cell).

\subsection{Statistical Analysis}

To test the experimental hypotheses, Condition (inhalation and exhalation) $\times$ Stimulus Intensity (high, middle, and low) ANOVAs were performed on the mean 
discrimination accuracy rates. According to Mizuhara and Nittono (2022), to compare the mean discrimination accuracy rates in the respiratory phases with a one-tailed $t$-test, Bayes Factor $\left(\mathrm{BF}_{01}\right)$ was also computed. $\mathrm{BF}_{01}$ greater than 3 was taken as moderate evidence for the null hypothesis over the alternative hypothesis, and $\mathrm{BF}_{01}$ greater than 10 was taken as strong evidence for the null hypothesis over the alternative hypothesis (Wagenmakers et al., 2018).

\section{Results}

\subsection{Data Exclusion}

Data from eight participants were excluded from the analysis according to the following criteria: two participants were excluded because they had a history of asthma; one participant was excluded due to technical failure in the respiratory recording; one participant was excluded because they fell asleep during the experiment; and four participants were excluded because one or more cells $(2$ tasks $\times 3$ stimulus intensities $\times$ 2 respiratory phases) contained less than 22 trials. Consequently, data from 28 participants were analyzed.

\subsection{Discrimination Accuracy}

Figure 1 shows the mean accuracy rates of each respiratory phase in the fear discrimination task. The mean accuracy rates and standard deviations for low-intensity stimuli were $60.9 \%(S D=10.6)$ and $62.3 \%(S D=9.9)$ in the inhalation-phase and in the exhalation-phase trials, respectively; for middle-intensity stimuli, the mean accuracy rates were $85.7 \%(S D=9.3)$ and $85.9 \%(S D=10.2)$ in the inhalation-phase and in the exhalation-phase trials, respectively; and for high-intensity stimuli, the mean accuracy rates were $95.9 \%(S D=6.1)$ and $96.4 \%(S D=7.2)$ in the inhalation-phase and in the 
exhalation-phase trials, respectively. A Condition $\times$ Stimulus Intensity ANOVA showed the main effect of stimulus intensity, $F(2,54)=278.95, p<.001$, Greenhouse-Geisser $\varepsilon$ $=.753, \eta_{\mathrm{p}}{ }^{2}=.912$. However, the main effect of condition and the interaction between condition and stimulus intensity were not significant, $F(1,27)=0.58, p=.455, \eta_{\mathrm{p}}{ }^{2}$ $=.021$ and $F(2,54)=0.23, \varepsilon=.927, p=.797, \eta_{\mathrm{p}}{ }^{2}=.008$, respectively. Moreover, the mean accuracy rates, collapsing all stimulus intensities, were $80.9 \%(S D=7.3)$ and $81.6 \%(S D=7.3)$ in the inhalation-phase and in the exhalation-phase trials, respectively. Bayesian paired $t$-test indicated moderate evidence for the null hypothesis, $\mathrm{BF}_{01}=8.16$. These results suggested that the respiratory phase did not affect the discrimination accuracy of choosing fearful faces over neutral ones. Therefore, the discrimination accuracy of emotional visual stimuli was similar for inhalation and exhalation.

Figure 2 shows the mean accuracy rates of each respiratory phase in the contrast discrimination task. The mean accuracy rates and standard deviations for lowintensity stimuli were $74.3 \%(S D=10.2)$ and $75.2 \%(S D=12.9)$ in the inhalation-phase and in the exhalation-phase trials, respectively; for middle-intensity stimuli, the mean accuracy rates were $87.5 \%(S D=10.1)$ and $88.9 \%(S D=9.2)$ in the inhalation-phase and in the exhalation-phase trials, respectively; and for high-intensity stimuli, the mean accuracy rates were $93.3 \%(S D=6.1)$ and $95.0 \%(S D=5.9)$ in the inhalation-phase and in the exhalation-phase trials, respectively. A Condition $\times$ Stimulus Intensity ANOVA showed the main effect of stimulus intensity, $F(2,54)=127.15, p<.001, \varepsilon=.793, \eta_{\mathrm{p}}{ }^{2}$ $=.825$. However, the main effect of condition and the interaction between condition and stimulus intensity were not significant, $F(1,27)=1.66, p=.208, \eta_{\mathrm{p}}{ }^{2}=.058$ and $F(2,54)$ $=0.07, p=.903, \varepsilon=.818, \eta_{\mathrm{p}}{ }^{2}=.002$, respectively. Moreover, the mean accuracy rates, collapsing all stimulus intensities, were $85.1 \%(S D=7.2)$ and $86.3 \%(S D=8.3)$ in the 
inhalation-phase and in the exhalation-phase trials, respectively. Bayesian paired $t$-test indicated strong evidence for the null hypothesis, $\mathrm{BF}_{01}=10.18$. These results suggested that the respiratory phase did not affect the discrimination accuracy of choosing highcontrast Gabor patches over low-contrast ones. Therefore, the discrimination accuracy of visual stimuli, in general, was similar for inhalation and exhalation.

\section{Discussion}

This preliminary study produced similar results to those seen in Mizuhara and Nittono (2022). Both studies strongly support the view that the effect of respiratory phase on discrimination accuracy of visual stimuli is not observed when people voluntarily adjusted the timing of stimulus presentation to their breathing.

\section{References}

Mizuhara, K., \& Nittono, H. (2022). Visual discrimination accuracy does not differ between nasal inhalation and exhalation when stimuli are voluntarily aligned to breathing phase. International Journal of Psychophysiology, 173, 1-8.

https://doi.org/10.1016/j.ijpsycho.2021.12.009

Schulz, A., Schilling, T. M., Vögele, C., Larra, M. F., \& Schächinger, H. (2016). Respiratory modulation of startle eye blink: A new approach to assess afferent signals from the respiratory system. Philosophical Transactions of the Royal Society B, 371, 20160019. http://doi.org/10.1098/rstb.2016.0019

Wagenmakers, E. J., Love, J., Marsman, M., Jamil, T., Ly, A., Verhagen, J., Selker, R., Gronau, Q. F., Dropmann, D., Boutin, B., Meerhoff, F., Knight, P., Raj, A., van Kesteren, E. J., van Doorn, J., Šmíra, M., Epskamp, S., Etz, A., Matzke, D., ... 
Morey, R. D. (2018). Bayesian inference for psychology. Part II: Example applications with JASP. Psychonomic Bulletin \& Review, 25, 58-76.

https://doi.org/10.3758/s13423-017-1323-7 


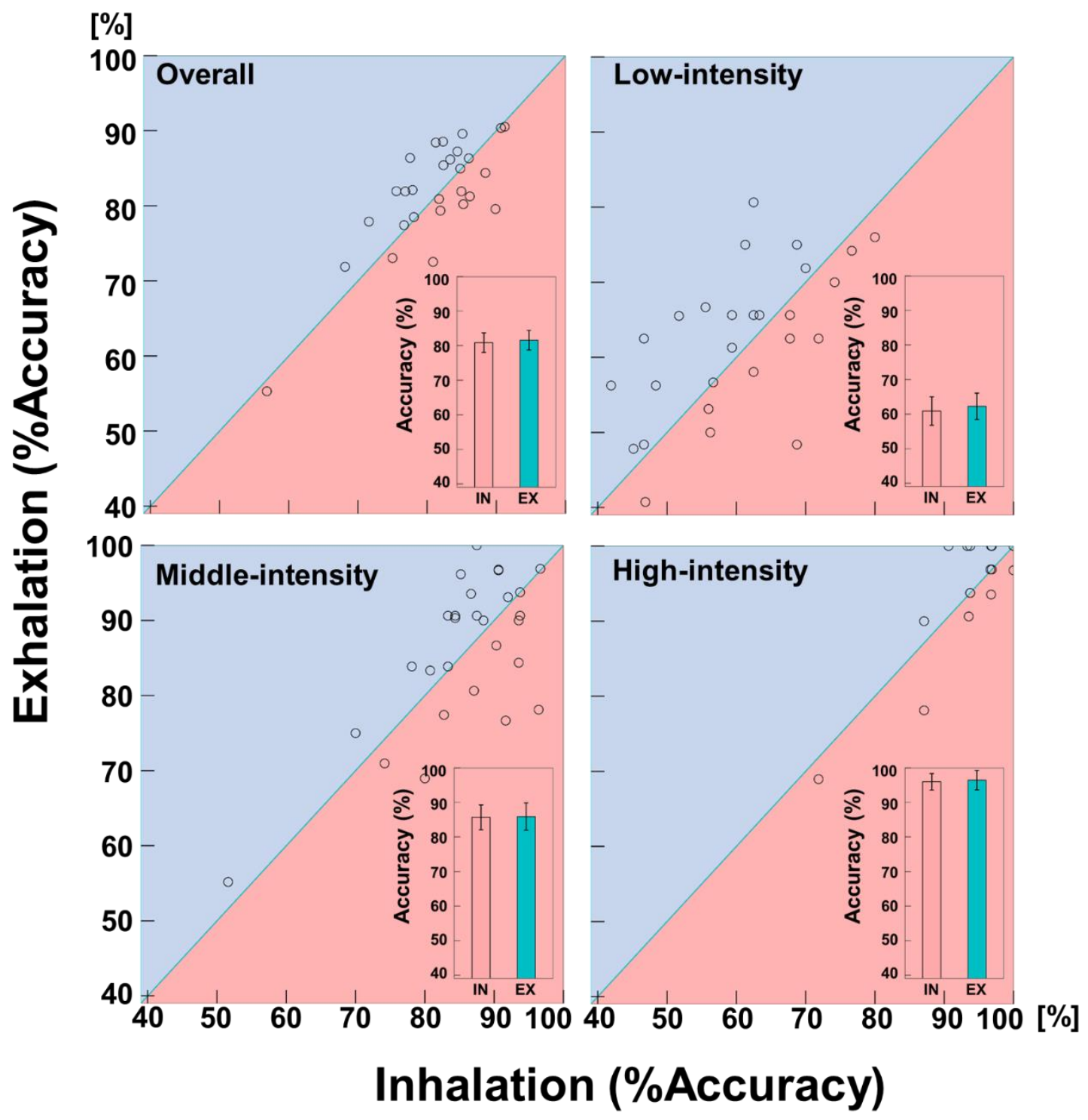

Figure 1. Scatter plots of the mean accuracy rate for each stimulus intensity as well as overall intensity for respiratory-phase trials in the fear discrimination task $(N=28)$.

Each participant's data corresponds to each point. Points below the diagonal line indicate that participants showed better performance in the inhalation-phase than in the exhalation-phase trials. Error bars of inset graphs indicate $95 \%$ confidence intervals. 


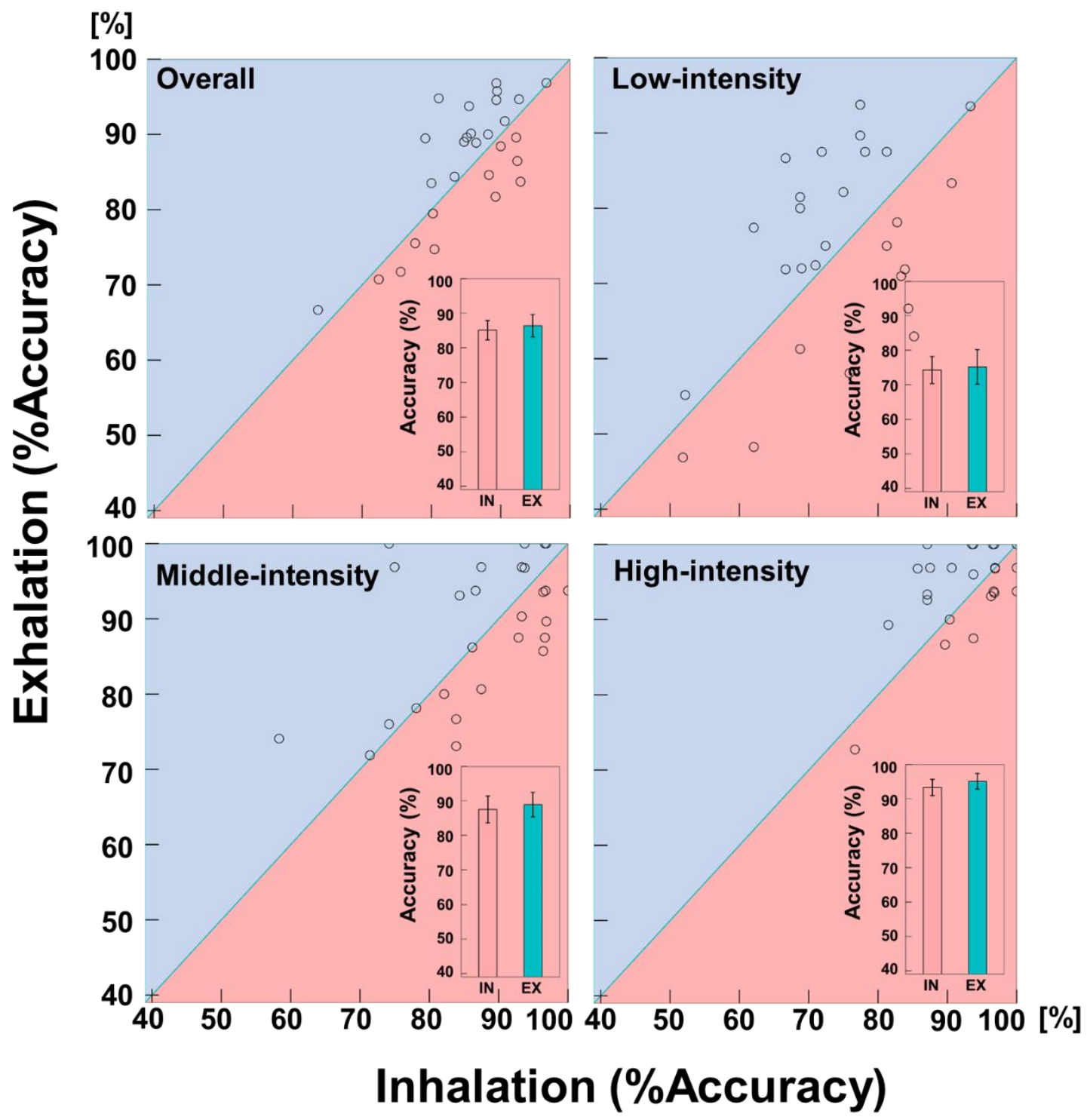

Figure 2. Scatter plots of the mean accuracy rate for each stimulus intensity as well as overall intensity for respiratory-phase trials in the contrast discrimination task $(N=28)$. Each participant's data corresponds to each point. Points below the diagonal line indicate that participants showed better performance in the inhalation-phase than in the exhalation-phase trials. Error bars of inset graphs indicate 95\% confidence intervals. 